\title{
Obsessive-Compulsive Disorder With Poor Insight and The Schizo-Obsessive Spectrum About a Clinical Case
}

Roberto Silva ${ }^{1}$, Antónia Fornelos ${ }^{1}$, Marta Roque ${ }^{2}$, Ana Margarida Ribeiro²

1 Psychiatry Resident, Centro Hospitalar de Trás-os-Montes e Alto Douro, E.P.E

${ }_{2}^{2}$ Psychiatry Assistant, Centro Hospitalar de Trás-os-Montes e Alto Douro, E.P.E

OBJECTIVES: The authors intend to discuss the co-occurrence of obsessive-compulsive and psychotic symptoms, as well as the relevance of a dimensional approach based on a schizo-obsessive spectrum. A clinical case is presented as a starting point to literature review and further discussion.

BACKGROUND AND AIMS: Obsessive-compulsive Disorder (OCD) is classically defined by the presence of recurrent thoughts and related behaviours described as intrusive and undesirable, with recognition by the patient of its pathologic quality. However, a minority of patients with OCD do not recognize the symptoms as ego-dystonic and have been described as having "poor insight". Poor insight in OCD patients has been associated with greater severity of symptoms, greater comorbidity, worse response to treatment and lower overall functioning.

RESULTS: M.S., male, only child, 21 years old, single, with 9 years of schooling, unemployed. Living with his parents, both with psychiatric history (father, 59, diagnosed with Schizophrenia and OCD; mother, 57, with Recurrent Depressive Disorder).

Initial contact with Mental Health services at the age of 13 for anxiety symptoms and worsening school grades. At 16 years old he began describing recurrent, intrusive, ego-dystonic thoughts related to self-destructive ideas and impulse phobia. The patient described fear of self-harmful behaviours such as the ingestion of poisonous liquids, pins or broken glass, and checking rituals characterized by the need to repeatedly verify the integrity of harmful items around him as well as induced vomiting to ensure he did not ingested any product. Organic evaluation was unremarkable and psychological evaluation showed he was of average intelligence. The patient was diagnosed with Obsessive Compulsive Disorder and medicated in the following years with different antidepressants (sertraline, escitalopram, fluvoxamine, paroxetine, mirtazapine, clomipramine) and antipsychotics (olanzapine, aripiprazole, quetiapine) in association with psychotherapy, with little improvement.

The years following diagnosis were marked by progressive social isolation and inability to maintain either school attendance or a job. The patient's insight into his symptoms became progressively vague and he started reporting self-referential ideas.

At 20 years old he was first admitted to a Psychiatry Inpatient Unit due to worsening symptoms and dysfunctional family environment. He was treated with a combination of paroxetine and haloperidol, with reduction in frequency of intrusive thoughts and greater control of checking rituals. Worsening of symptoms after discharge required new admissions to this unit, for a total of six admissions during the same year, with improvement followed by relapse after discharge. The dysfunctional familiar dynamics, with all the members symptomatic, was suspected to reinforce doubt and insecurity, negatively influencing recovery.

In the current year, the patient was one of the first to attend Day Hospital recently implemented in our Department, and to benefit from an intensive daily treatment in outpatient setting. This treatment modality was focused on improving autonomy and developing social and self-management skills, with good results.

CONCLUSIONS: This case illustrates the diagnostic and therapeutic challenges of patients with obsessive-compulsive and psychotic symptoms. It reminds us that insight should be viewed as a fluid concept and not as an "all or none" dichotomy, and the distinction between obsessive and delusional thoughts may not always be clear. This seems to agree with what has been described in literature. The repeated relapses after discharge may suggest non-compliance and/or worsening of symptoms in response to a dysfunctional environment. This, in association with family history, highlights how biological and social factors may contribute to the clinical picture. Comorbidity of psychosis and OCD is more common than previously thought, and some authors have suggested a new diagnostic entity as a more fitting label than that of comorbidity. "Obsessive Psychosis", "Atypical $O C D$ " or "Schizo-obsessive Disorder" are some of the names being proposed. Others have postulated that it should be viewed as part of a "Schizo-obsessive Spectrum", with OCD on one end and Schizophrenia on the other, and that patients may transition along this spectrum throughout their history, with obsessions giving way to overvalued ideas, and these to delusions. This seems to fall in line with recent trends to shift from categorical to dimensional diagnostic labels. Overall, this underscores the complexity of mental processes, the need for further research in the area, as well as the necessity of an individualized approach aimed at meeting each patient's needs.

Attademo L. et al, "Schizophrenia and obsessive-compulsive disorder: from comorbidity to schizo-obsessive disorder", in Rivista di Psichiatria 2012; 47(2): 106.

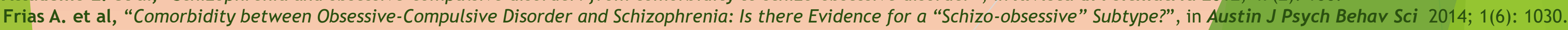
Kamaradova D. et al, "Correlates of insight among patients with obsessive compulsive disorder", in Act Nerv Super Rediviva 2015; 57(4): 98. Oulis P. et al, "Diferential diagnosis of obsessive-compulsive symptoms from delusions in schizophrenia: A phenomenological approach", in World J Psychiatr 2013; 3(3): 50. Scotti-Muzzi E., Saide O., "Transition from Obsession to Delusion in Schizo-obsessive Disorder: A Case Report and Literature Overview", in Innov Clin Neurosc $2018 ; 15(7-8)$ : 23. Zinc M., "Comorbid Obsessive-Compulsive Symptoms in Schizophrenia: Insight into Pathomecanisms Facilitates Treatment", in Advances in Medicine $2014 ; 317980$. 